\title{
Apical Implant Beveling for Management of Fenestration Defects
}

Bruna Tanello, D.D.S and Rodrigo Neiva, D.D.S, M.S.

University of Florida College of Dentistry - Department of Periodontology

\section{Abstract}

The undercut of the anterior maxilla often hinders placement of maxillary anterior implants. This anatomical feature may result in implants placed with excessive buccal angulation, which may prevent the use of screw-retained restorations. In order to promote adequate access for screw-retention, this angulation promotes thinning of the apical end of the osteotomy against the buccal wall, which may cause dehiscence or fenestration defects after osseointegration and bone remodeling. ${ }^{1}$ These defects may cause discomfort to the patient, or become source of infection, which may result in implant failure. ${ }^{2}$

\section{Background and Aim}

The aim of this presentation is to discuss treatment options for dehiscence defects caused by excessive palatal angulation of dental implants placed in the premaxilla for screw-retained restorations.

\section{Methods and Materials}

Ten consecutive patients were treated with a combination of apical beveling of the implant body, followed by disinfection of any exposed implant surface with citric acid for two minutes, and subsequent guided bone regeneration utilizing layers of bone cortical and cancellous allografts (Symbios, Dentsply) and longlasting absorbable barrier membranes (Perioderm, Dentsply). ${ }^{3}$
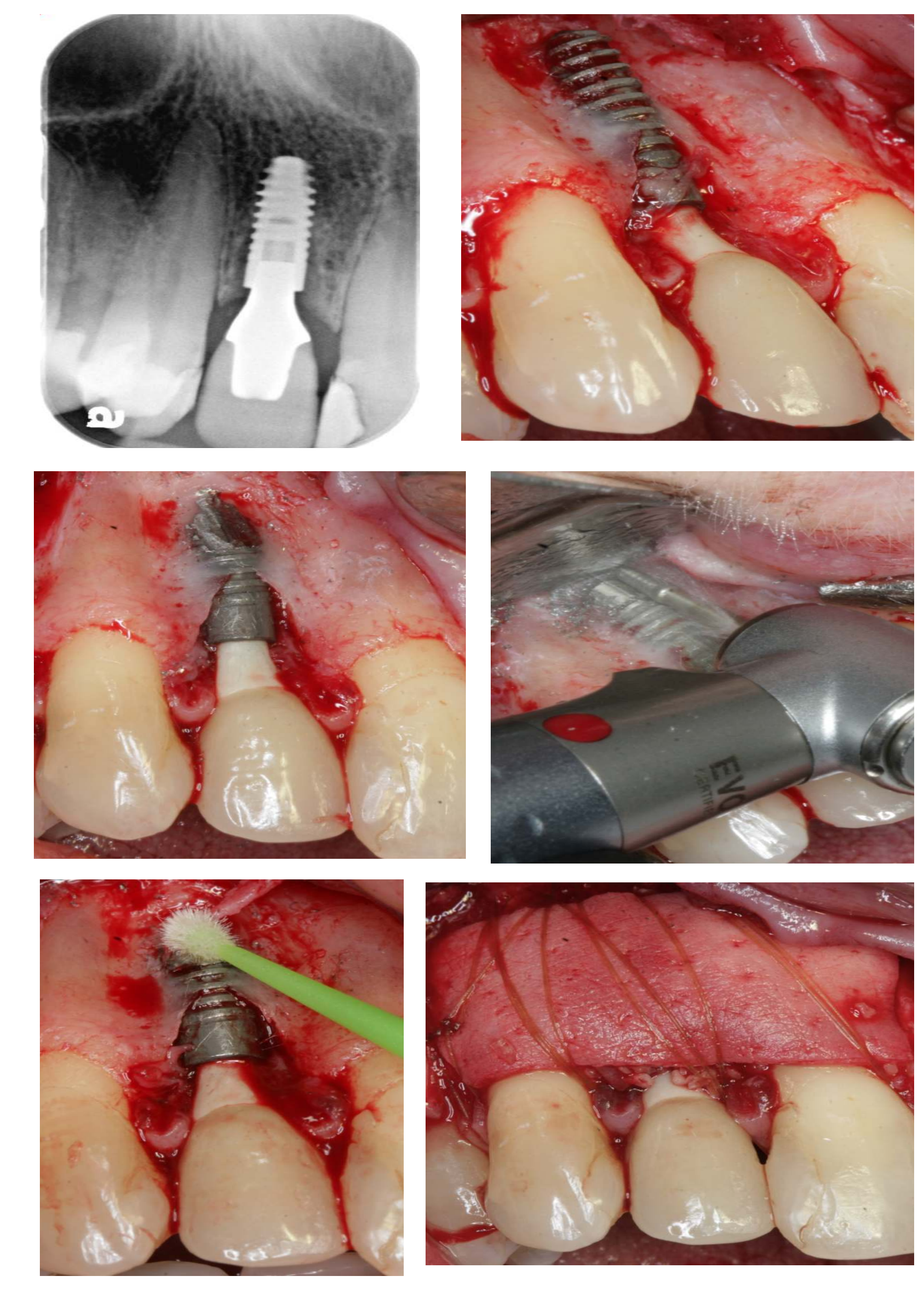

This material and GBR technique selection aimed to promote hard and soft tissue augmentation. These cases were reevaluated at two weeks, 3 months, 6 months, and 12 months. $\mathrm{CBCT}$ imaging was obtained at 12 months to confirm bone fill over the previously existing defects.

\section{Results}

All implants experienced absence of symptoms post-treatment. No discomfort was reported by any of the patients. Clinically, all implants appeared to be stable and continue to be in function. No signs of additional drainage or fistula formation were detected. CBCT evaluation at 12 months confirmed these findings. Signs of bone fill and defect resolution were observed. Treated sites experienced a mean gain of buccal plate thickness of $2.45 \mathrm{~mm}$ (Range: $1.8 \mathrm{~mm}-4.2 \mathrm{~mm}$ ) All patients were satisfied with treatment outcomes.

\section{Conclusions}

This clinical observation demonstrated that apical beveling of maxillary anterior implants showing dehiscence type of defects, associated with guided bone regeneration, is a viable option for management of this kind of late implant complication that is often seen in clinical practice. Future studies should evaluate the longterm success of this treatment option.

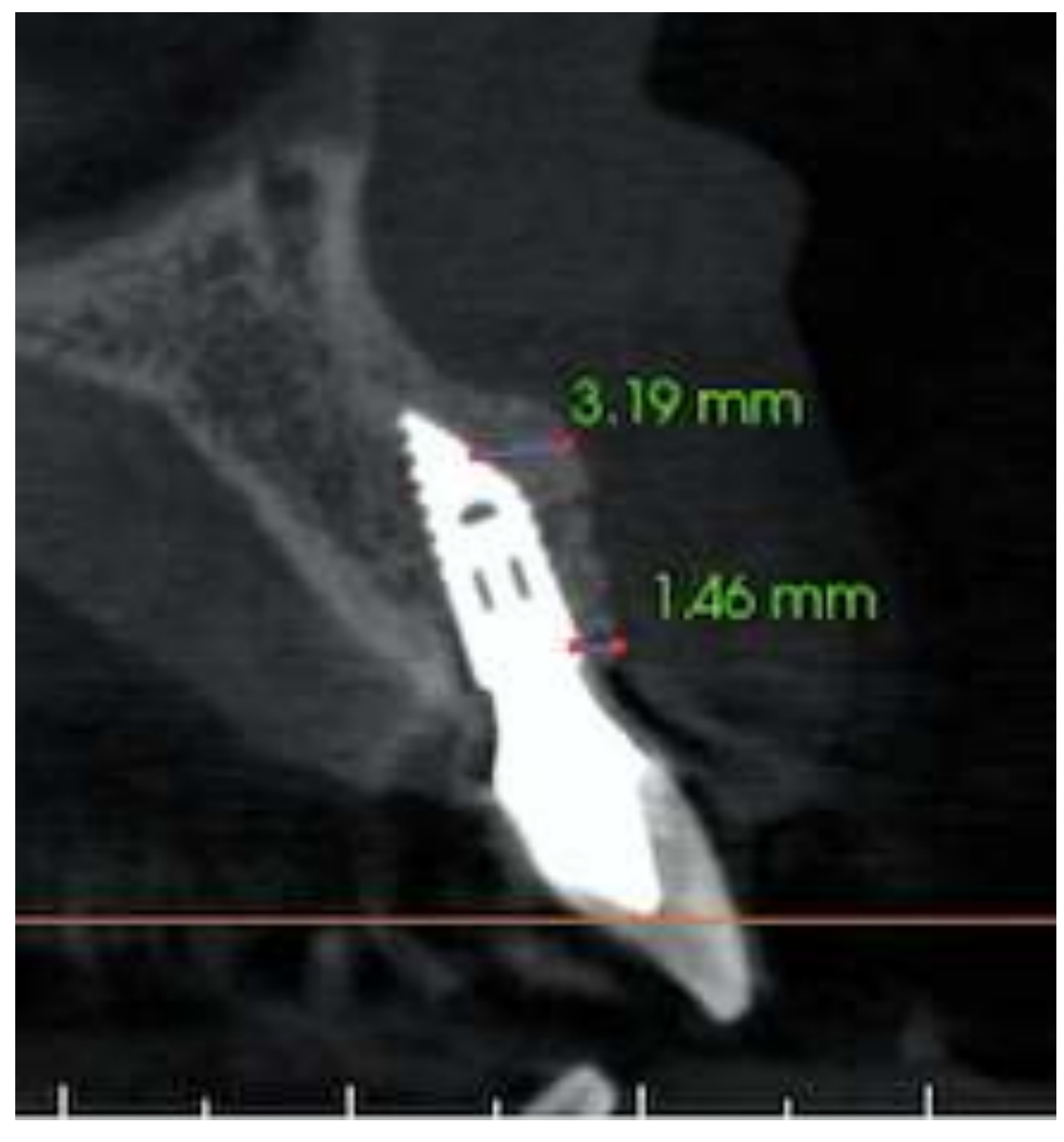

\section{References}

1 - Chan $\mathrm{HL}$ et al. Incidence of implant buccal plate fenestration in the esthetic zone: a cone beam computed tomography study. Int J Oral Maxillofac Implants. 2014 Jan-Feb;29(1):171-7. 2 - Chiapasco M, Zaniboni M. Clinical outcomes of GBR procedures to correct peri-implant dehiscences and fenestrations: a systematic review. Clin Oral Implants Res. 2009 Sep;20 Suppl 4:113-23. 3 - Wang HL, Misch C, Neiva RF. "Sandwich" bone augmentation technique: rationale and report of pilot cases. Int J Periodontics Restorative Dent. 2004 Jun;24(3):232-45. 